\title{
ظواهر اللغة العربية والصعوبات التي يواجهها الناطقون بغيرها
}

\author{
أفريجون أفندي \\ جامعة السلطان الشريف قاسم الإسلامية الحكومية رياو، اندونيسيا \\ aprijonefendi@yahoo.com
}

\begin{abstract}
ملتخص
اللغة هي ذاتية للحضارة وكذلك اللغة العربية. لقد لعبت العربية منذ فترة طويلة دورها في الحضارة الإنسانية. وحاليا، بذلت الأمم المتحدة باللغة العربية كواحدة من اللغات العالمية الرئيسية، حتى بr بلدا في جميع أنحاء العالم استخدامها كلغة رسمية. في عصر العولم، واجهت العديد من التحديات في تعليم اللغة العربية للناطقين بغيرها. وفي الواقع أن اللغة العربية هي اللغة التي لا يمكن فصلها عن الإسلام، ولكن العديد من المسلمين غير قادرين على التواصل بهذه اللغة. بشكل عام، فإن المشاكل في تطوير اللغة العربية للناطقين بغيرها هي عدم وجود البيئة اللغوية الداعمة، وصعوبة للحصول على المعلمين المختصين والمهنيين بها، وصعوبة في إيجاد منهج جيد ومختبر. ومن مساهمة لعلاج تلك المشكلة منها هيئة البيئة اللغوية، وإعداد المعلمين، والمناهج الدراسية، وطرق التدريس الجحديدة والمتنوعة، وجود مشاركة المؤسسات الدولية في تطوير تعليم اللغة العربية، والدول العربية التي تساعد على تمويل البحوث في تطوير هذا العمل.

الكلمات الئيسية: الحضارة، المشكلات، الصعوبة، والحلول
\end{abstract}

ظواهر اللغة العربية والصعوبات التي يواجهها الناطقون بغيرها -

لقد تطور تعليم اللغة العربية للناطقين بلغات أخرى تطورا ملموساً في الآونة الأخيرة. وتعهد بتعليمها الكثير من البلاد الإسلامية وغيرها، فافتتح الكثير من المعاهد والمدارس والمراكز لتعليمها، وأولى المتخصصون جل اهتمامهم في سبيل توفير الفرصة أمام كل من رغب في تعلم العربية فاهتموا بوضع الكتب الميسرة وأعدوا الأماكن والمعلمين، ولكن الباحث لاحظ أن هناك بعض الصعوبات اللغوية تواجه الدارسين للغة العربية الناطقين بغيرها، فاستعن بالله، وأخذ في جمع غالبية تلك الصعوبات اللغوية واقتراحات لكيفية علاجها .بعد التجربة التعليمية للطلاب في قسم تدريس اللغة العربية بكلية التربية والتعليم جامعة السلطان الشريف قاسم الإسلامية الحكومية من خلال محاضرات خحاصة بتعليم اللغة العربية لهم فقد وجد الباحث 
أن هؤلاء الطلاب يفقدون الكثير من المحاضرات العلمية الخاصة بالدراسة بسبب فقدان اللغة، والازدواجية اللغوية التي يعتمد عليها بعض الأساتذة أثناء الشرح، و إلى جانب الاختلافات الصوتية التي يقعون فيها، فالاخرافات الصوتية تعد أكثر من بقية المستويات اللغوية الأخرى.

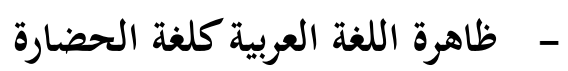

اللغة هي مرآة الحضارة الإنسانية، ليست استثناء من اللغة العربية. ومع ذلك، ليست لكل

اللغات تلعب دورا هاما للحضارة البشرية، فضلا عن الدور الذي لعبته اللغة العربية، أن لديها قوة من حيث الوصف والتجريد، والدقة من معنى (الدقة الدلالية)، وتمكن تشكيل الكلمات المشتقة (الاشتقاق)، وتفاصيلها رسخ وجودها في المسار التاريخي للحضارة الإنسانية.' ووصلت ذروتا مع ظهور الإسلام في

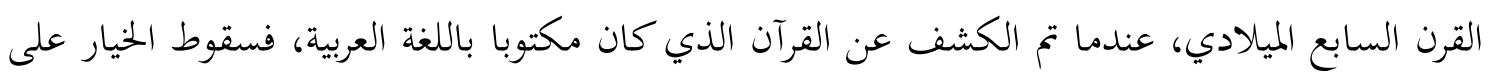
اللغة العربية كوسيلة وصول الوحي من الله عز وجل هو شهادة على قوة أثر هذه اللغة بالنسبة اللغات الأخرى في تسجيل الرسالة الربانية للفكرة البشرية، وأها مليئة بالمفردات التي تحتوي على الفلسفة، والتاريخ،

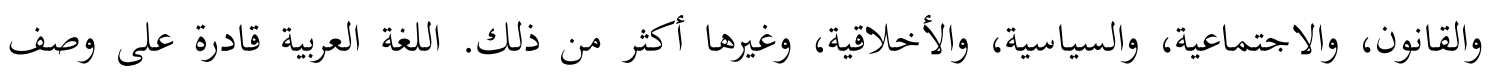
وحي من الله تعالى إلى سبيل الحق، والتفاصيل، والتأكيد حتى لا يمكن بها خاطئ في ترجمته وتفسيره. ولكن. خلاف عن ذلك، أن بعض الكلمات في اللغة العربية تحتوي على معان واسعة مقيدة بمقاصدها، وأحيانا في كلمة واحدة تحتوي على اثنين من المعاني، سواء حرفيا أو تمثيليا. وفي هذه الحالة، كانت اللغة العربية أكثر ملائمة وموثوقة بالمقارنة مع اللغات الأخرى من أجل توفير اليقين في المعان الواضحية (النصية) والضمنية (السياقية) من خلال المعاني في القرآن كدليل البشرية الذين يعيشون في مختلف الدول والقبائل. إن اللغة العربية لديها تاريخ طويل من التطورات على ساحة الدين والحضارة، سواء في البحال الثقافية واللغوية والسياسية والاجتماعية والاقتصادية والقانونية في مختلف البلدان العربية ومناطق أخرى، بحيث كانت اللغة العربية هي واحدة من اللغات الرئيسية للغالبية العظمى من السكان في العالم، ووجودها

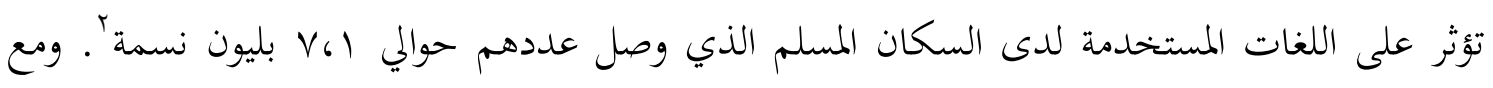
ذلك، كانت تطور اللغة العربية تتقدم مرة وتتأخر مرة أخرى جنبا إلى جنب مع الأحياء وسقوط الحضارة الإسلامية التي تمتد على الفترة الزمنية الطويلة من عهد الدولة الأموية، والدولة العباسية، ودولة المماليك، حتى الدولة العثمانية التي انتهت سيطرتا على العالم في عام ع بو (م)، وبعد ذلك أصبحت الدول العربية

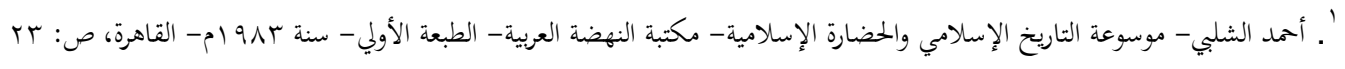
' ب أمد شلبي - المرجع السابق، ص: 10 
تحت استعمار البريطانية والفرنسية. وفي عهد استعمار الإنحليزية والفرنسية لم تكن اللغة العربية تستضيف أرضها الأصلية، وحينئذ استخدمت اللغة الإبحليزية والفرنسية في جميع الشؤون الإدارية الحكومية.

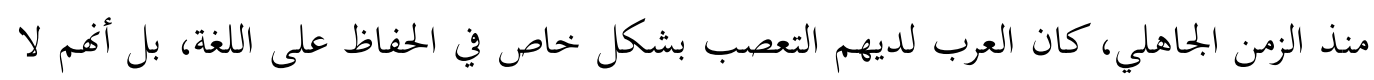
يريدون أن يؤثرو عليهم اللغات الأجنبية. واللغة العربية نفسها ليست لغة جامدة وميتة التي لا تقبل التطور. وفي عهد الدولة الأموية اصبحت اللغة العربية كاللغة الرسمية للدولة حتى حدث تطبيق نظام التعريب و يجب لهب أن يكون كل شيء العربي. وفي ذلك الوقت، كانت اللغة العربية لديها نموا سريعا للغاية لأن العديد من

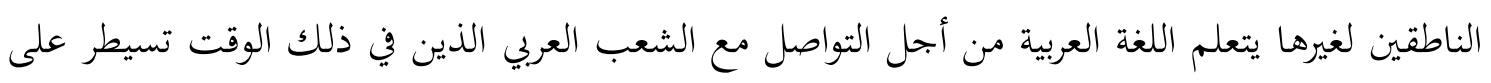
الحضارة العالمية.

وفي الواقع، أن اللغة العربية هي جزء لا يتجزأ من حياة المسلمين. ولذلك، تعلم واتقان اللغة العربية تعتبر من احتياجات المسلم. ووجود اللغة العربية أمر ضروري لبناء شخصية المسلم في تحسين نوعية

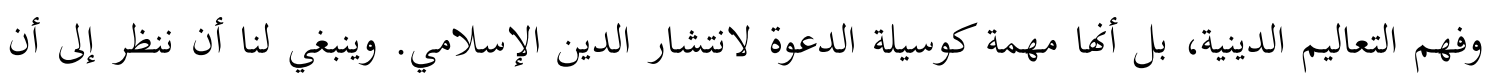

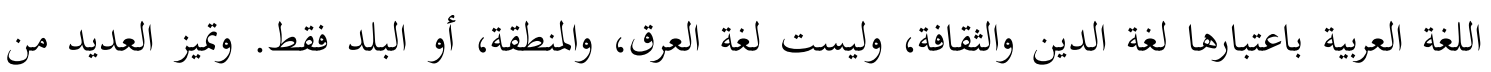
القادة والعلماء المسلمين الذين ليسوا من العربيين كمثل الغزالي، والبخاري، والبيروني، وابن سينا، والرازي، والكندي وغيرهم، وهم يستوعبون اللغة العربية كجزء من لغة الدراسات الإسلامية التي يتعمقون هما. وفي عهد العولمة، هناك التحديات المعاصرة التي تواجه اللغة العربية وتفرض على الأمة الإسلامية والعربية بأسرها أن تعظم من جهودها للارتقاء بالعربية وبمكانتها تعليميا وحضاريا ودوليا، ونستشهد هنا بما

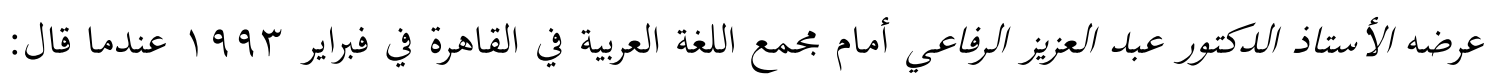

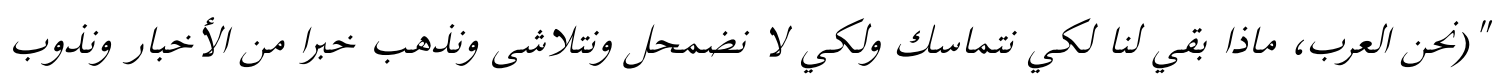
كما ذابت من قبلنا أمم فقلت شخصيتها السياسية؟ لم بيق لنا إلا اللغة. فقل تكالب علينا الغزو الثقافي من كل جانب، يعمل دائما ليل نهار لكي يقوض بلغتنا، لغة القرآن، ويكولها إلى أمشاج يسهل ابتلاعها وتكوينها على النحو الني يريله، هو يلرك تماما أنه قوض اللغة قوض الدعامة الأساسية في كياننا، وسمّل علينا بعل ذلك أن يقوض بعلدها كل الدعامات الأخرى لتنهار واحد تلو الأخرى، وبنلك لن تتوم لنا قائمة ولا تجمعنا رابطة)"، وبما ذكره الدكتور نبيل على أن من تحديات تواجه اللغة العربية والثقافة الإسلامية

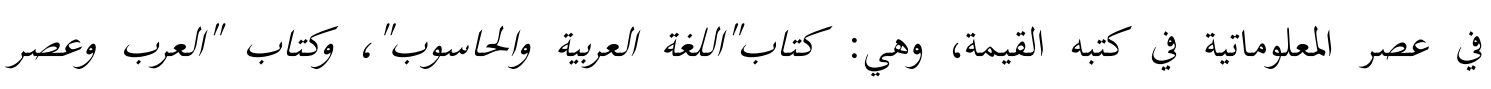

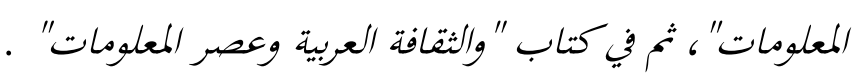

- - ماهرة اللغة العربية كاللغة الدولية

وقد اعترفت اللغة العربية باعتبارها لغة عالمية ويتم استخدامها واحدة من اللغات الرسمية الأساسية للدبلوماسية في منتدى الأمم المتحدة، بل كان بعض الدول غير العربية في العالم مثل ماليزيا اعترفت اللغة 
العربية باعتبارها لغة مهمة التي ينبغي دراستها، حتى تكون حكومة الدولة تعطي الفرصة لانتشار الكتابة العربية في الأماكن العامة فيها. وفي بلدنا إندونيسيا، على الرغم من عدم وجود الكتابة العربية انتشاريا بالتساوي، ولكن في هذه الأرض الملايو "علامة صفراء" منذ عهد رَجَا عَلِي حَاجي كانت الكتابة العربية لها مكان خاص في قلوب الحكومة والبحتمع. هناك الكثير من الجهود التي تبذلها الشعب العربي من أجل تنهيض وتطوير اللغة العربية بعد

$$
\text { استقلالهم من احتلال بريطانيا وفرنسا، ومن بين الجهود المبذولة هي: }
$$

r ـ وفي عام 0ـ9 ا تم تأسيس جامعة الدول العربية التي تلعب دورا رئيسيا في تطوير اللغة العربية كاللغة

الدولية.

ץ. وفي عام به 90 (، بناء على اقتراحات وزراء التعليم في البلدان أعضاء جامعة الدول العربية أنشئت

$$
\text { جمعية بحمع اللغة العربية. }
$$

ع. وفي عام 907 1، تم تنظيم المؤتمر لجمعيات اللغة وإنشاء لجنة التنسيق بين جمعيات اللغة العربية في جميع أنحاء البلاد العربية، ولجنة الصياغة التنمية للغة العربية، ولجنة الإعداد وتشكيل المصطلاحات

$$
\text { وبتحميع القواميس. }
$$

ه. وفي عام • ب7 19، أسست معهد الدراسة والبحث وتطوير اللغة العربية.

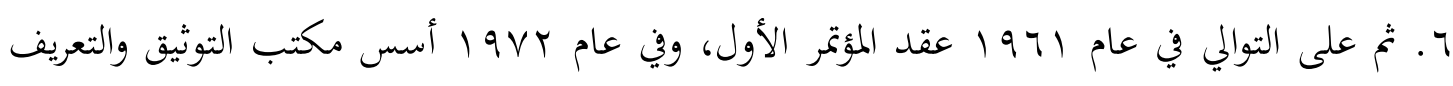

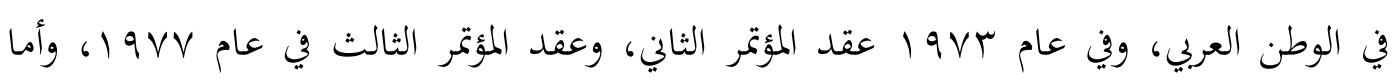

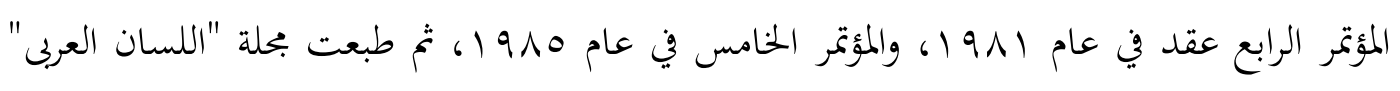
وذلك من أجل استهلاك بحموعة متنوعة من الأفكار حول اللغة و وضع السياسات في تطوير اللغة

$$
\text { العربية. }
$$

V. تنظيم دورة ألعاب التضامن الإسلامي (ISG)، وهذه الدورة تنعقد مرة في كل أربع سنوات، وأها واحدة من الجهود الرئيسية لرفع مستوى اللغة العربية كلغة التي توحد المسلمين في جميع أنحاء العالم. وين عصرنا الحديث، هناك تطورات هامة حول دراسة تطوير اللغة العربية، على الرغم من أن علم الصرف والنحو وقواعد للغة العربية التي وضعت بالفعل لا يمكن تغييرها بسهولة. وأما الدراسة والبحث في

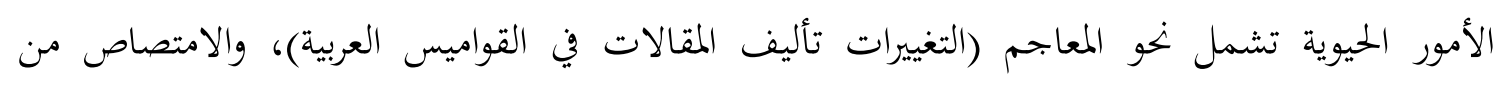

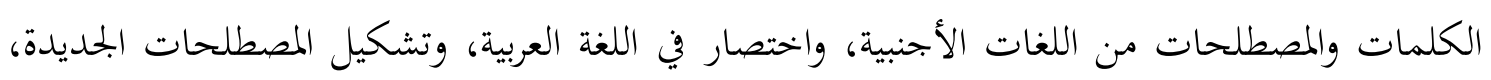

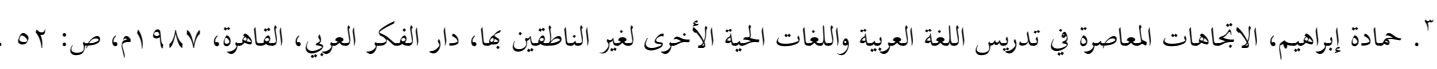


والترجمة الصوتية، وغيرها. وهذه الدراسات بتري تطويرها تدريجيا، بل أغها ليست المستخدمة في الدراسة من الدراسات العربية والإسلامية فحسب، وإنما أيضا لأجل التنمية في بحالات أخرى، مثل علم الآثار، وفقه اللغة، والتاريخ، والأدب، و والتعليم وغيرها.

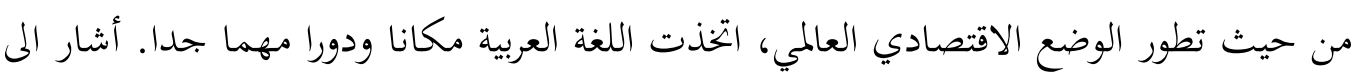
ذلك أهميتها المتزايدة في منطقة الشرق الأوسط كمركز للطاقة والثروة المعدنية في العالم مع أن أغلبية سكاها يتحدثون باللغة العربية. وجدير بالمعرفة، أن العديد من الناس في العالم مهتم بهذه المنطقة ونود أن يفتح خطوط الاتصال مع الدول فيها، وفي الفكرة والموقف أهم بحاجة إلى إتقان اللغة العربية، لأها "المدخل" من التواصل بين الثقافات التي مهلت الطريق لتثبيت العلاقات الاقتصادية والسياسية، حتى كثير من الأجانب الذين يعملون في منطقة الشرق الأوسط يستوعبون اللغة العربية، و على سبيل المثال: كان سفير الصين وروسيا وألمانيا، وهولندا في جمهورية السودان قادرين على التحدث باللغة العربية بطلاقة. وهم يدركون أن اللغة العربية إلى جانب اللغة الإبحليزية هي الشرط الرئيسي لنجاج الاتصال والدبلوماسية فضلا

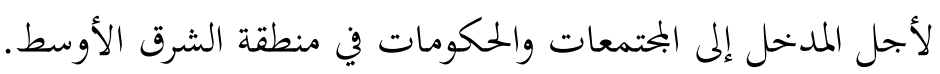

كانت عالمية اللغة ترجع إلى اتفاق علماء اللغة على عالميا على أها وسيلة الاتصال الأولى بين المحتمعات وأداة لنقل الحضارات المختلفة بين الشعوب. والإقبال على تعليم العبية من غير الناطقين بها لقي اهتماما كبيرا لكوها لغة القرآن. وكذلك كان الإقبال على تعلم العربية له أهداف كثيرة منها بجانب الأهداف الدينية فهناك أيضا الأهداف الثقافية والاقتصادية. و من أهم ما يشكل الصعوبات اللغوية هو التداخل (interference) بين اللغة العربية ولغاتم الأصلية في الجوانب الصوتية والنحوية والدلالية والكتابية. وأبرز الفروع اللغوية التطبيقية المسئولة بالدرجة الأولى عن تعليم اللغة العربية للناطقين بلغات أخرى هو علم اللغة التطبيقي، فهو الآن من أبرز الفروع التطبيقية لعلم اللغة الحديث (Modern Language) فتعليم اللغة العربية للناطقين بغيرها يعتمد اعتمادا أساسيا على معطيات تعليم اللغات الأجنبية المستمرة من نتائج الدراسات اللغوية النفسية (psycholinguistic studies) والتطبيقات التربوية، وهي علوم حديثة، نشأت في الدول الغربية، وازدهرت فيها خدمة للغاتم وبخاصة اللغة الابحليزية، ونخن المسلمين لا بنح حرجا من الاستفادة من هذه العلوم ونشرها. وأن علم اللغة التطبيق (Applied Linguistics) أحد فرعي علم اللغة (Linguistics) (General Linguistics) في الغالب، ثم الكلمات فالعبارات فالجمل. ولابد أن تتكامل هذه الحلقات في نسق معين، فالأصوات المحردة لا قيمة لما ولا دلالة ما لم تضف إلى بعضها لتكوين كلمة والكلمة تكتسب معناها المحدد عندما تنضم إلى كلمات أخرى لتكون العبارة أو الجملة. 


\section{- المشكالات في عملية تعليم اللغة العربية}

مسألة التعليم هي مسألة أساسية في بناء المجتمع وتطوره وتقدمه أو تراجعه وفساده ودماره. إن

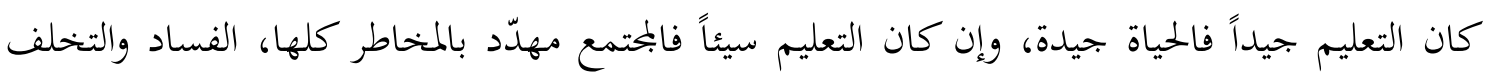
والاهيار. وفي بحث هذا الموضوع قسم الباحث المشكلات في عملية تعليم اللغة العربية إلى قسمين، وهما:

\section{أ. المشكالوت العامة.}

والمشكلات العامة في التعليم تكمن في المعلم والمنهج وطريقة الإيصال، فاختيار المعلم لمراحل التعليم كافة في الدول الإسلامية بتحري على غير ما يجب أن تكون عليه، والاتساع في التعليم فرض على وزارات التربية تحاوناً وتساهلاً في قواعد الاختيار، فيسألونه في العلم والحياة والثقافة، ويختبرون قوة شخصيته وسرعة بديهته وأشياء يطول بياها، فكيف نسلّم اليوم أجيالنا لمعلمين أو معلمات بعضهم جاء إلى هذه المهنة مقهوراً بلا زاد ولا معرفة؟ والمنهج تدخلت في وضعه الوزارة تحقيقاً لأهداف الدولة والبحتمع، ولكنّ الذين اختارو ذلك لم يكونوا على قدر المسؤولية، فهؤلاء يجب أن يكونوا من العلماء الذين لا يؤمنون إلا بالبحث العلمي وسيلة وسبيالًا لوضع منهاج يحقق الأهداف، ويتدرج بالمعلومات.

\section{ب. ب. المشكلات الخاصة.}

وطريقة التوصيل مرتبطة بالمنهج والمعلم والاستعداد لدى المدرسة أو الوزارة لإغناء المدرسة بكل جديد متطور لتكون المدرسة مكاناً محبباً للطلاب الذين يسعون إليه، لأنه بيته أو لعله أجمل من بيوقهم. ويبين الباحث هذه المشكلات الخاصة في تعليم اللغة العربية على النقاط التالية:

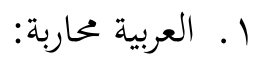

يحاربها المتربصون بالأمّة العربية، فقد زُيّنت لأولي الأمر وللمجتمع العلوم التجريبية، فالعصر عصر العلوم التكنولوجية، وكان ذلك على حساب العلوم الإنسانية وأولها وأغناها اللغة العربية، فلم يعد معلم العربية المعّلم الأول، بل تراجع ليكون أثره ضعيفاً، وما عرف هؤلاء وأولئك أن العلوم التجريبية ذاتما سيضعف مستواها لأن الأساس فيها هو اللغة، وازدادت الأمور سوءاً في بعض الدول العربية حين أخذوا بنصائح العولمة، فأهلوا العربية، وأحلّوا الابحليزية محلّ الصدارة.

$$
\text { ץ . الإيهام بصعوبة اللغة العربية: }
$$

أُدخل في روع الناس أن اللغة العربية لغة صعبة، عصيّة على التعلم، هي لغة قديمة، وقواعدها بائدة، لا علاقة لها بالحداثة، ولا يمكن لها التعبير عن علوم العصر، وأنا أزعم أن اللغة

؛ . فتحي على يونس ومحمد عبد الرؤوف الشيخ، "المرجع في تعليم اللغة العربية للأجانب: من النظرية إلى التطبيق"، مكتبة وهبة، القاهرة،

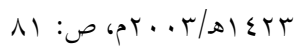


العربية أسهل من كلرّ اللغات إن كان المحال بحال المقارنة بين السهولة والصعوبة، ولكنّ إضعاف

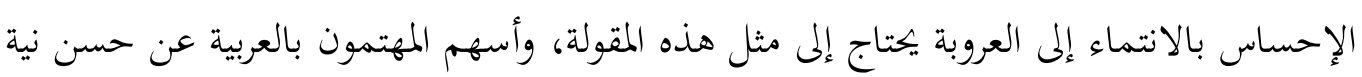

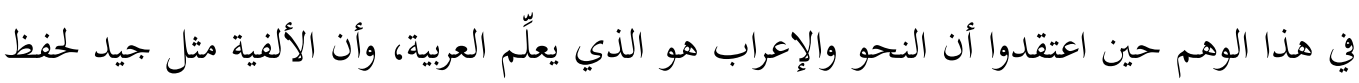

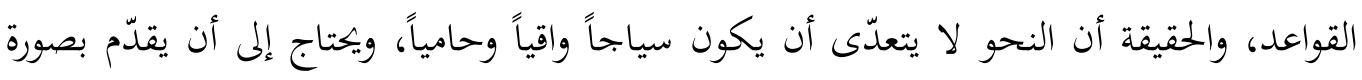

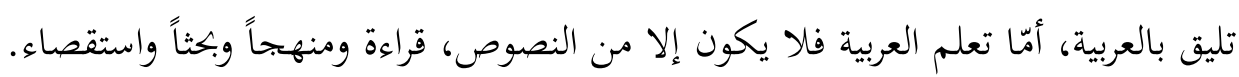

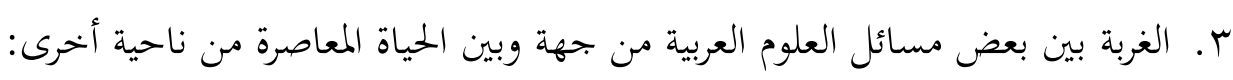

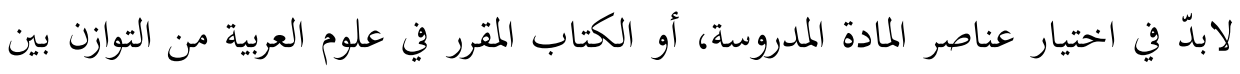

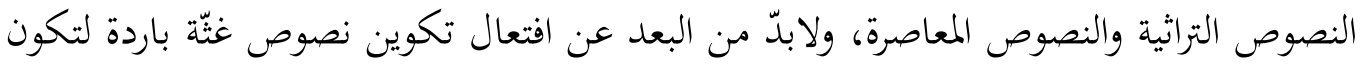

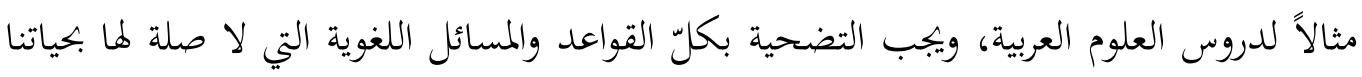

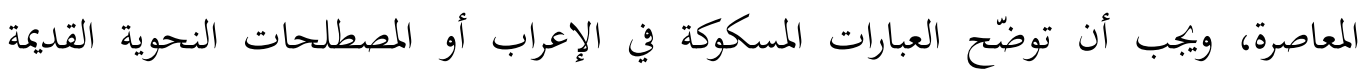

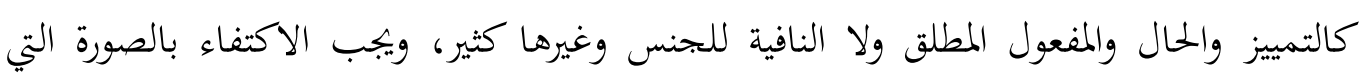

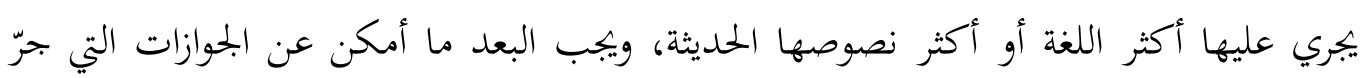

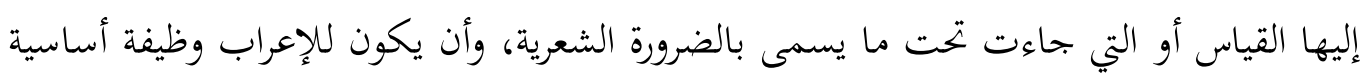
هي إدراك المعنى، ويجب التساهل في الشكل، وقبول التسكين مبدئياً. ع. اختيار النصوص:

النصّ النثري المختار يجب أن يتميّز بعنصري التشويق والإثارة، وأن يتم اختياره وفقاً

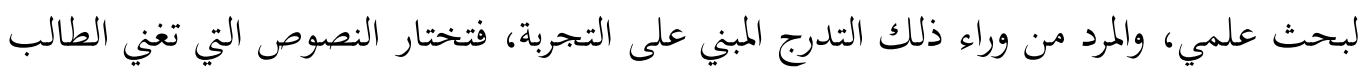

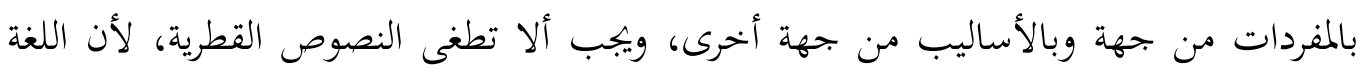

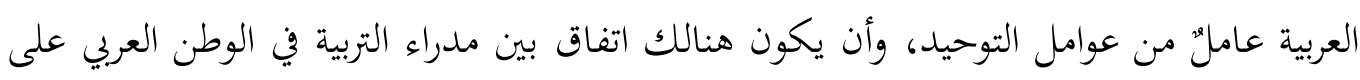
ذلك.

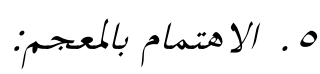

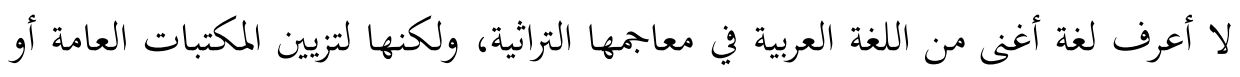

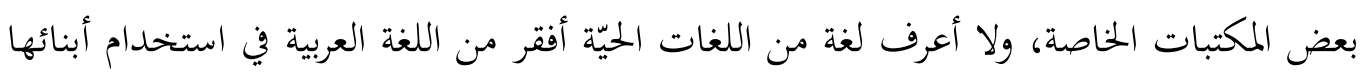

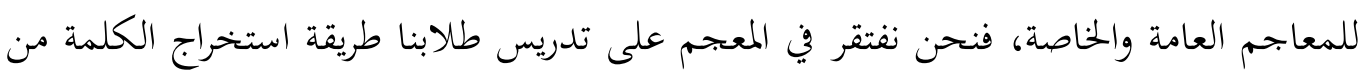

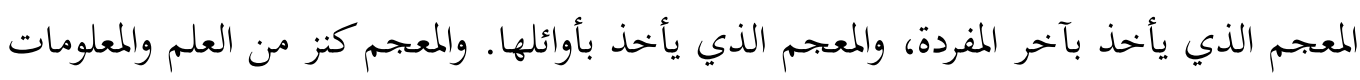

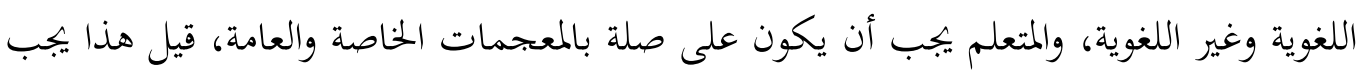
أن تكون الثقافة المعجمية ثقافة عامة، ولابدّ من التوجه إلى هذا اللون من التأليف لأنه مازال قلئلياً 
في الوطن العربي. نحن في حاجة إلى معجم معاصر يعنى بلغة الحياة التي نهياها حتى تقوم الصلة بيننا وبينه، فالصلة اليوم مقطوعة بالمعاجم القديمة لأنه لا فائدة منها.

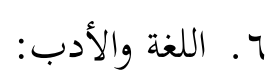

العلاقة أساسية بينهما، والمزج أو الخلط بينهما يمكن أن يؤدي إلى مشكلة تعليمية، فأنا اقترح أن يتعلم الطالب في مرحلة التعليم الأساسي الأولى القراءة والكلام بالفصيحة، ثمّ يتدرب في مرحلة التعليم الأساسي الثانية على قضايا النحو والصرف والمعجم وغيرها من خلال نصوص فيها متعة وتشويق وفائدة، أمّا الأدب وقضاياه فيجب أن يترك للمرحلة الثانوية مع تذكير دائم بما كان

$$
\begin{aligned}
& \text { من قواعد علوم العربية. } \\
& \text { V. التعبير: }
\end{aligned}
$$

لا أعرف شكوى أكثر مرارة من شكوى التلاميذ والطلاب حين يكلفهم المعلم أو المعلمة بكتابة موضوع يعبرون فيه عن أمر ما، فلا المدرس يعرف الطريقة التي يوصل فيها الطلاب طريقة الكتابة، ولا الطالب قادر على الإبداع والابتكار لأنه لم يدرب على ذلك. ودرس التعبير أو محاولة المتعلم التعبير عن شأن من شؤونه الخاصة أو من شؤون الحياة شيء له أهمية خحاصة،

$$
\text { وهو إحدى مهارات العربية ولعله أهمها. }
$$

وأما حلول من المشكلا المذكورة، على المدرس لابد أن يختار موضوعاً كتب فيه هو أو غيره،

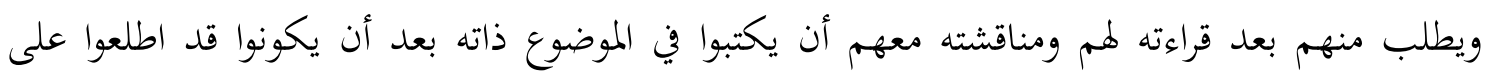
نموذج أمامهم، ومع ذلك فالبحث في موضوع التعبير يحتاج إلى دراسة أعمق وأشمل وإلى بتريب مستمر بهر حتى نصل إلى الطريقة المثلى لتعليم هذه المهارة. والخطوة التطبيقية للخروج من هذه المشكلات لابد أن نفهم دور العناصر التالية، وهي:

\section{ا ـ ا ازدواجية اللغة:}

ليست هذه المشكلة خاصة بتعليم اللغة العربية، فأغلب اللغات إن لم يكن كلها لديه ازدواجية في اللغات بل ازدواجيات، ولكنها تصبح مشكلة عصية على الحل حين يتم التعليم باللغة المحكية، وكأن التعليم بالفصحى خاصة في درس اللغة العربية، بل زعم الباحث أن تعليم العربية لايتم إلا باللغة المحكية، وزعم أيضاً أن بعض أساتذة الأدب والنحو في الجامعة يعلمون بالعامية أو اللغة المحكية. والحقيقة أن التعليم بالفصحى ليس مقصوراً على مدرس اللغة العربية فحسب، بل هو فرض واجب على كل من يعلم، فالفصحى وسيلة التعبير السهلة والميسورة، وعلى من لا يستطيع ذلك أن يبتعد عن هذه المهنة، وهذا الأمر يجب أن يكون قراراً سياسياً أو وزارياً ترعاه الدولة. 


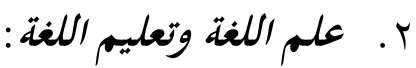

إن علم اللغة لا يعلم اللغة، إنه علم بقوانين اللغة، وفرق بين اللغة وقوانينها، فالقوانين

سياج يحمي، ولكن التعلم لا يتمّ إلا من خلال الممارسة والدربة، والعدوى، فالقراءة الصحيحة

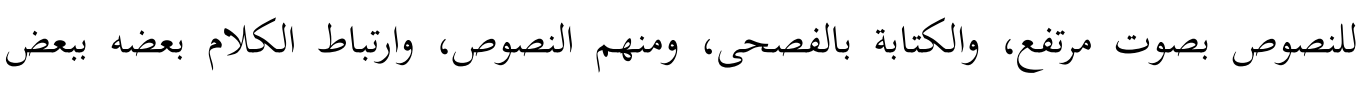
يؤدي إلى التعليم، ولابد من تسهيل هذا العلم، والتسهيل لا يتم إلا بتغيير القوانين، فالقانون ثابت، ولكن التسهيل يتم بإعادة النظر بالتعبير عن هذا القانون أو في اختيار القوانين اللغوية التي

$$
\text { تشكل لغة الحياة المعاصرة. }
$$

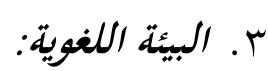

لابد من العمل على تنقية البيئة المكتوبة من الكلمات الأجنبية أو العامية، لقد شاعت

في حياتنا الكتابة بالعامية أو بالأجنبية (والكتابة بالأجنبية اعتراف بالضعف لألفيك والتخلف) للدعايات

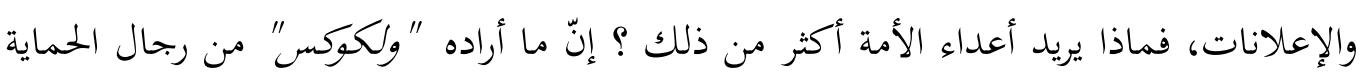
الانحليزية في جمهورية مصر العربية، وهو يريد أن تكون الكتابة في تلك الدولة باللسان العامية المصرية، وذلك سيتحقق بعد نضال في معاداته لأكثر من قرن من الزمان، ولذا نحن بحاجة إلى يلى البيئة المويدة لممارسة اللغة العربية الفصحى، وإنّ البيئة اللغوية النظيفة تساعد على تعليم وتعلم العربية الفصحى بدون العامية.

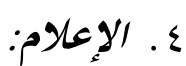

كان لابدّ للإعلام المسموع والمقروء والمرئي أثر بالغ في اتساع انتشار العربية الفصحى، فالعربية الفصحى التي يستخدمها الإعلام ولاسيما في نشرات الأخبار على ما فيها من أخطاء

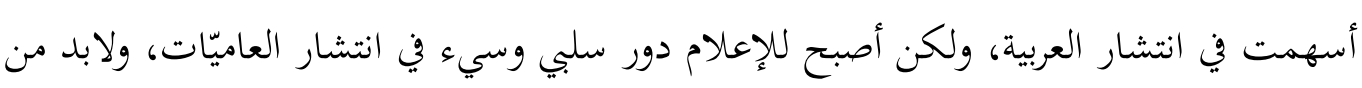
إعادة النظر في هذا الأمر، فاستخدام الفصحى البسيطة في المسلسلات يؤدي إلى انتشار العامية في الوطن العربي والعالم الإسلامي، ويؤدي إلى انتشار اللغة العربية الفصحى أهم عامل للتوحيد.

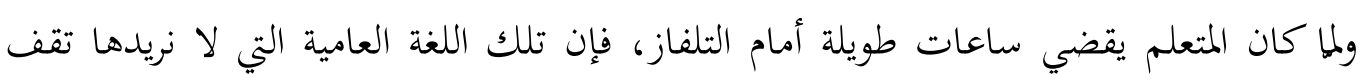
عائقاً أمام تعليم اللغة العربية الفصحى.

\section{- الصعوبات في تعليم اللغة العربية للناطقين بغيرها}

إن الصعوبات التي تواجه المدرسين في تعليم اللغة العربية للناطقين بغيرها ليست كلها صعوبات

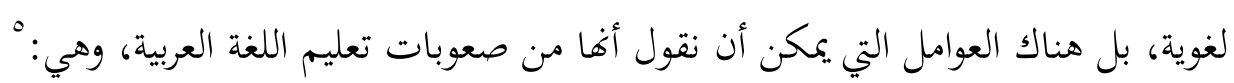

" عبد الرمن كادوك، تكنولوجيا التعليم: الماهية والأسس والتطبيقات العملية، دار المفردات للنشر والتوزيع، الرياض، الطبعة الأولى، ... بم، ص: VI TV 
أولا: الصعوبات التي تراجع إلى الدارسين أنفسهم، وذلك للأسباب التالية:

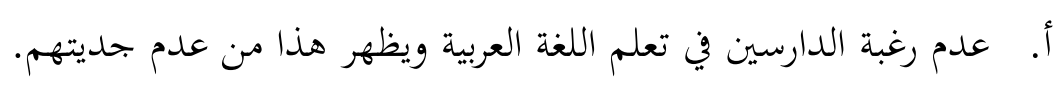
ب. عجز بعض الطلاب عن استيعاب الجحديد خاصة إذا تزايد الكم المقدم. ت. بعض الدارسين كبار السن. ث. كثرة مشاغل الدارسين وعدم تفرغهم للدراسة. ج. عدم حفظ بعض الدارسين القرآن الكريع.

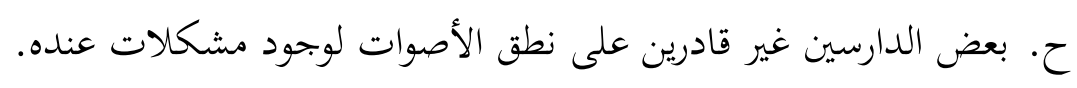
خ. عدم أداء التدريبات التي يكلف بها الدارس في البيت.

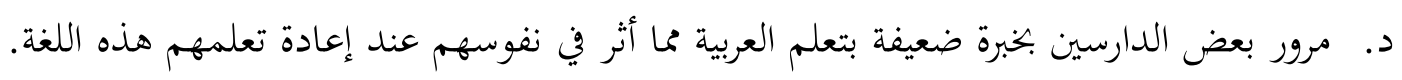

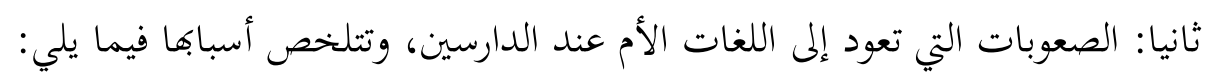

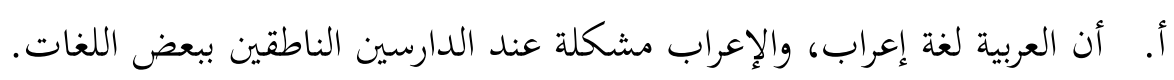

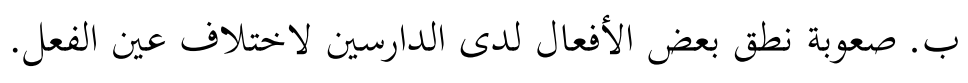

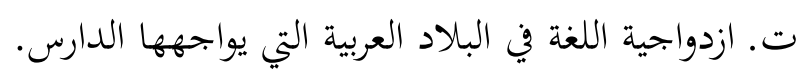
ث. لا يستطيع الدارس التفريق بين الأصوات المرققة التي لها أصوات مطبقة من نفس نواتل نوعها.

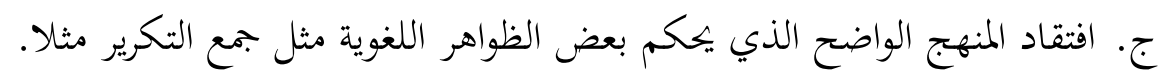
ح. وجود تشابه بين الحروف سواء في نطقها أو في كتابتها. خ. كثرة المترادفات في العربية تؤودي إلى الاضطراب التعليمي لدى لفى الدارسين.

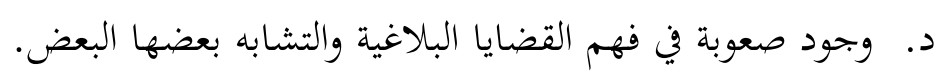

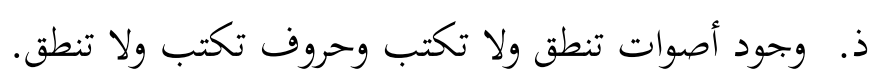
ر. عدم ضبط الحروف العربية بالشكل في بعض المواد المطبوعة.

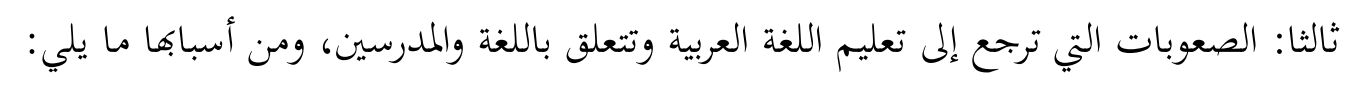

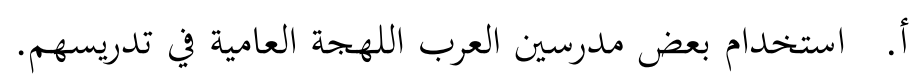

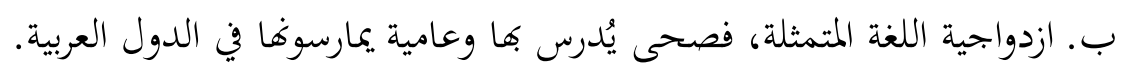

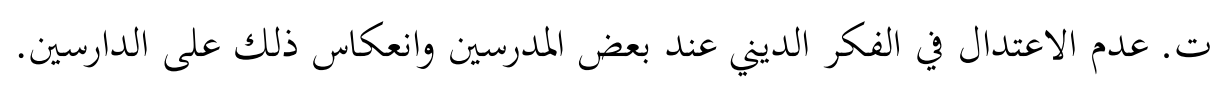

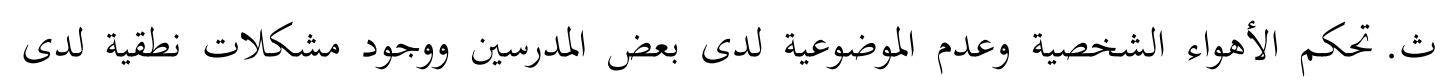

\section{بعضهم.}

ج. قلة العنصر النسائي المتخصصات في التدريس في هذا البحال. ح. عدم العمل بروح الجماعة التي تساعد كثيرا في حل الصعوبات اللغوية لدى لدع الدارسين. 
خ. عدم كفاءة بعض المدرسين واستخدامهم طرقا تقليدية في تعليم العربية كلغة ثانية. د. ندرة المتخصصين في تعليم العربية للناطقين بغيرها وافتقاد التدرج في عرض المعلومة اللغوية. ذ. توحيد طريقة التدريس بين تعلم أهل اللغة والوافدين عليها.

ر. عدم الكفاءة في استخدام اللغة الوسيطة، فأحيانا تستخدم بكثرة عندما لا يحتاج إليها ولا تستخدم عندما يحتاج إليها.

ز. عرض المادة اللغوية داخل الكتب بطريقة تقليدية وعدم إبراز جماليات النصوص اللغوية. س. قد تضم الكتب الكثير من الكلمات الصعبة التي لم تسبق دراستها في المستويات السابقة للدارس. ش. عدم الاتفاق العلمي على منهج لغوي تربوي، يراعي فيه الأسس المنهجية لوضع مثل هذه الكتب . n ص. عدم الاهتمام من قبل علماء اللغة بوضع مواد لغوية تعليمية جاهزة لمعامل اللغات، وعدم وجود مؤسسات تعليمية للغة العربية بصورة كافية. ض. توحيد الكتاب المدرسي لدى المبتدئين بالرغم من اختلاف مستوى الدارسين المبتدئين، فمنهم من عنده خلفية لغوية عربية ومنهم من يفقد هذه الخلفية بل تنعدم عندهم أي معرفة باللغة العربية وأنظمتها. ط. عدم الالتفاف إلى ميول الدارسين في القراءة، فاختيار المحتوى اللغوي عند تأليفه يجب أن يسبقه مسح ميداني لاستكشاف ميول الدارسين التي تقدم لهم هذه الدراسة.

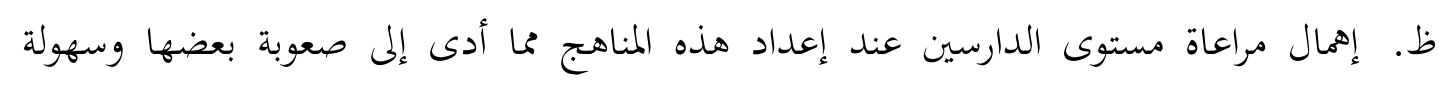
البعض الآخر.

ع. عدم اشتمال المناهج اللغوية على نظام ممارسة اللغة في مواقف حية والمتابعة والتقويم. غ. عدم توفر كتب للقراءة الإضافية (Supplementary readers) و ووسائل تعليمية في بحال تعليم العربية للناطقين بغيرها.

ف. عدم الدقة في تحديد المستوى اللغوي للدارسين عند بدء التحاقهم بمعاهد تعليم العربية لغير

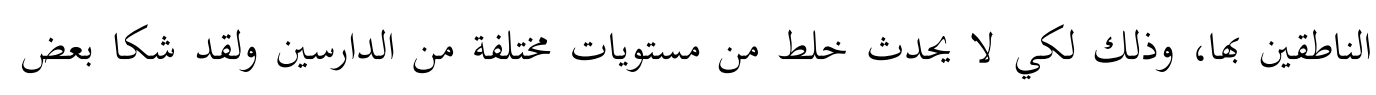
المعلمين في أحد معاهد تعليم العبية أن الدارسين في المستوى المبتدئ يتفاوتون في مستواهم اللغوي ومهاراهم التي يجيدوفا، فمنهم من يتكلم العربية إلا أنه لا يعرف القراءة، ومنهم من يقرأ بعض الكلمات إلا أنه لا يستطيع الكلام بالعربية، ومنهم من يفهم العربية بسهولة إلا أنه عاجز عن الكلام بها أو قراءقا وهكذا. 
ق. عدم توظيف إمكانات تكنولوجيا التعليم الحديثة الموجودة في مؤسسات تعليم العربية للناطقين بغيرها.

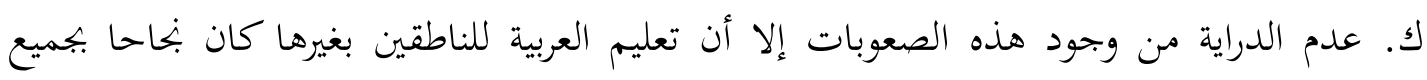
المقاييس لقوة الدافع لدى الكثير من المستعملين للُّة.

\section{- - سبل التغلب على صعوبات تعليم اللغة العربية للناطقين بغيرها}

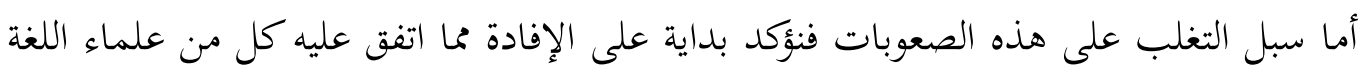

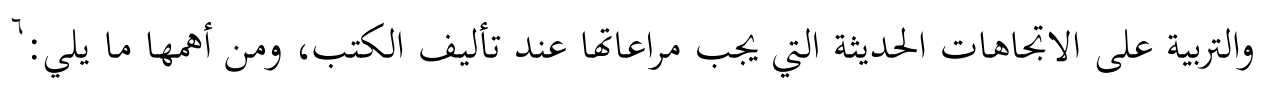

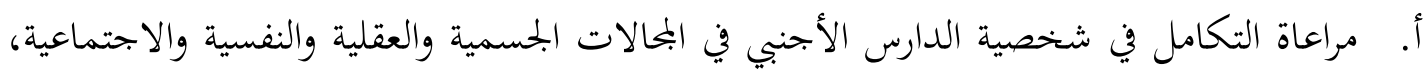

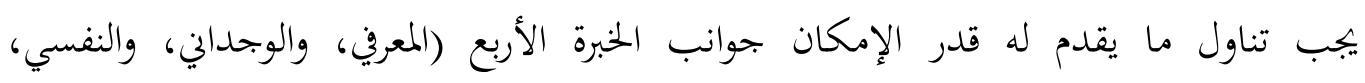

$$
\text { والحركي). }
$$

ب. مراعاة التكامل اللغوي بين المهارات الأربع (القراءة، والاستماع، والكلام، والكتابة) في المعالجة أداءً وتقويما.

ت. مراعاة طبيعة اللغة بوصفها تراكيب تصير بالممارسة سلوكا لغويا، مع انعقاد النشاط اللغوي

$$
\text { الشفهي أساس في تنمية قدرات المتعلمين الأجانب اللغوية. }
$$

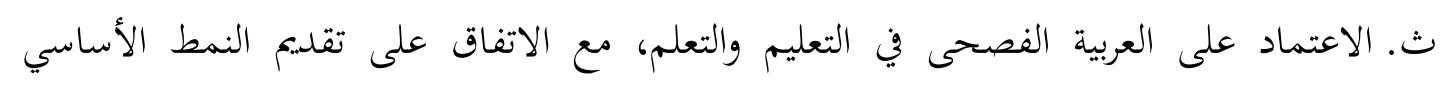
للدراسة الصوتية والنحوية والصرفية.

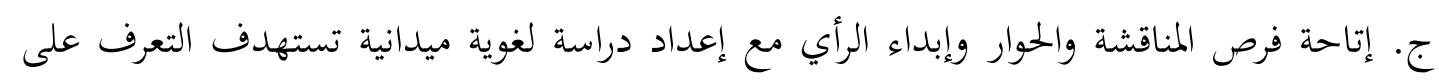
الحاجات اللغوية للدارسين.

ح. يراعى تقديم اللغة التي تناسب مستوى الدارسين الأجانب مع مراعاة التدرج في المستوى اللغوي

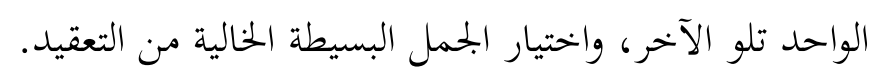

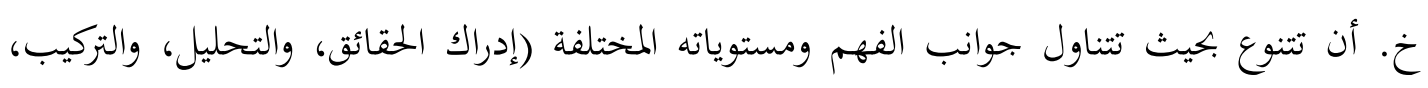
والتعليل، وإدارة التسلسل).

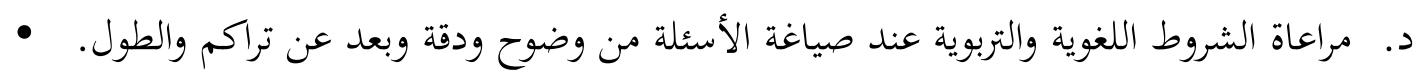

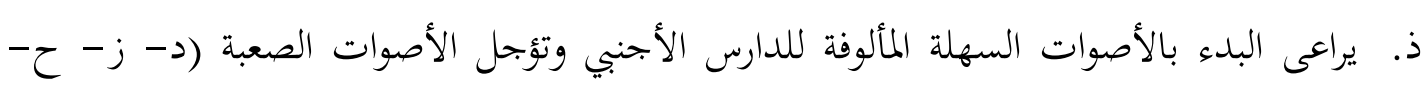
خ- ش-ص- ض- ط- ظ- ع- غ- ق)، مع مراعاة التدرج في الأصوات الصعبة كلما أمكن. 
ر. أن يعتني في المقدمة الشفوية خاصة وفي دروس الكتابة عامة بتقديم النطق الصحيح والواضح، والكال وتقديم التدريبات السمعية والنطقية الكافية لإكساب الدارسين الصحة والسرعة في النطق،

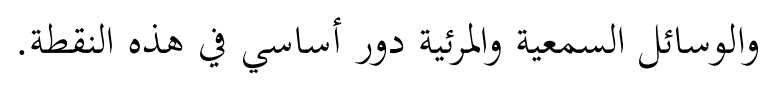

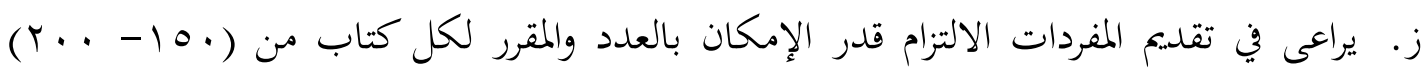

كلمة حسبما يتفق عليه كل من علماء اللغة والتربويين.

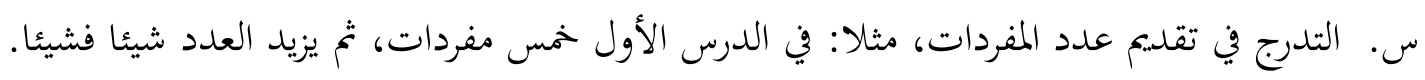

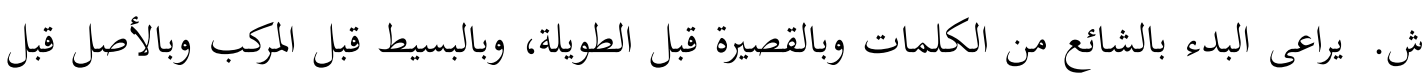

النوع.

ص. مراعاة ضبط المفردات بالشكل وبيان معنى المفردة، وتكرار المفردات الجحديدة عن طريق وضعها

$$
\text { في جمل مفيدة بصورة متوازنة. }
$$

ض.الانتباه إلى عدم تقديم القواعد أو المصطلحات النحوية بصورة مباشرة، وتعرض القواعد بطريقة

$$
\text { وظيفية في التراكيب اللغوية. }
$$

ط. نبدأ بالأسهل ثم الصعب فالأصعب في القواعد، وكذلك نبدأ بالإثبات قبل النفي وبالماضي قبل المضارع والمعلوم قبل المجهول والقياسي قبل الشاذ، والمذكر قبل المؤنث وهكذا.

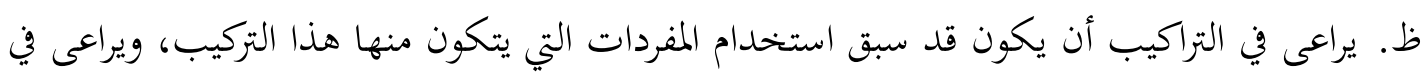

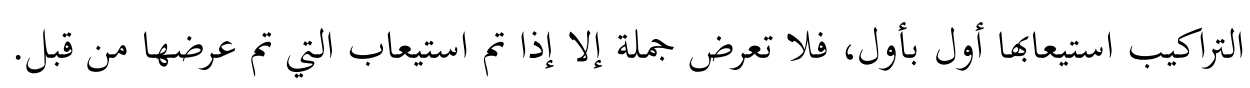

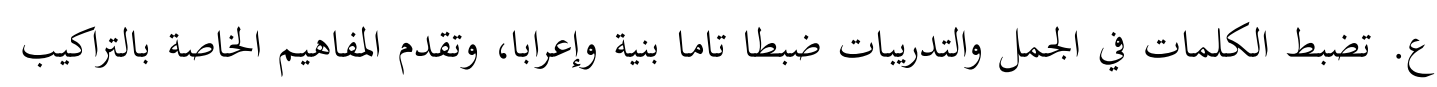

$$
\text { بطريقة الحوار قدر الإمكان. }
$$

غ. عمل معتم خاص بكل كتاب، بحيث بتمع المفردات التي تم عرضها في داخل المختوى التأليفي

$$
\text { وتثبيت في قوائم آخر الكتاب. }
$$

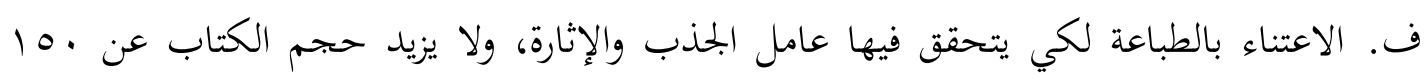
صفحة من الورق متوسط الحجم، والاستفادة قدر الإمكان بالتكنولوجيا الحديثة في هذا المحال.

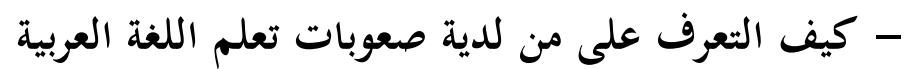

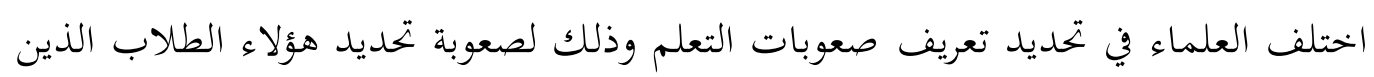

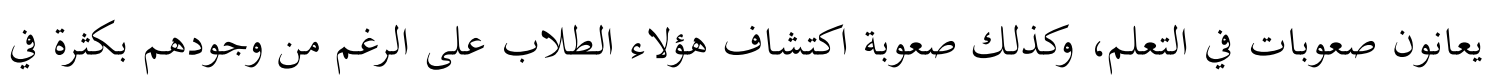

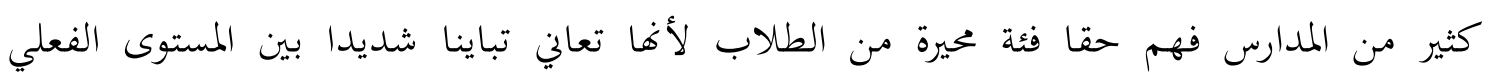

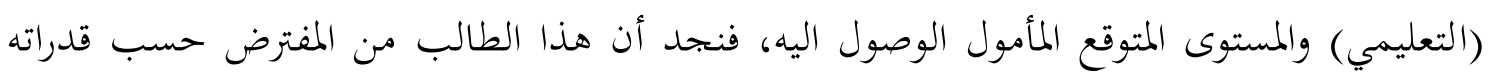

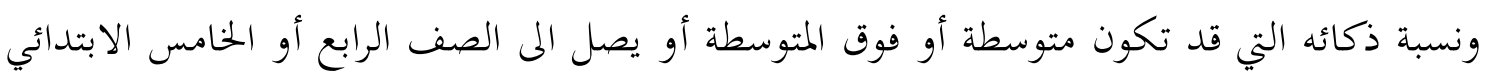


في حين أنه لم يصل الى هذا المستوى. ولأجل سهولة الفهم على هذ التعرف فرتب الباحث بحثه على النقاط التالية: أولا: فمن هم الطلاب النين يعانون صعوبات التعلم؟ النعالية

هم طلاب الذين لايعانون اعاقة عقلية أو حسية (سمعية أو بصرية) أو حرمانا ثقافيا أو بيئيا

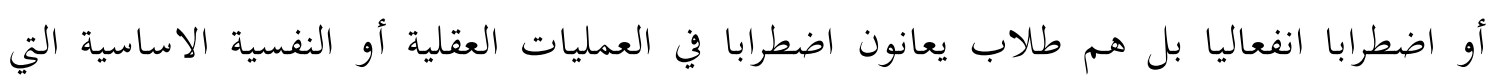

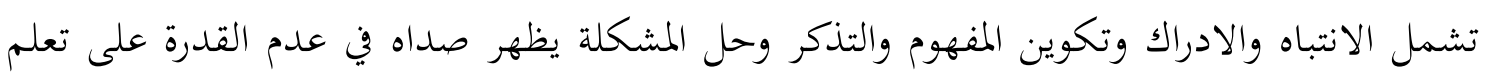

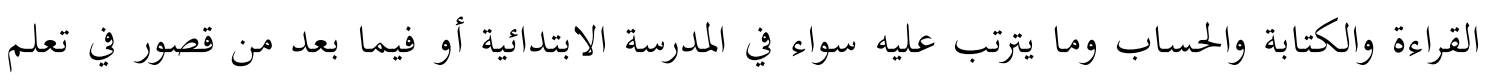

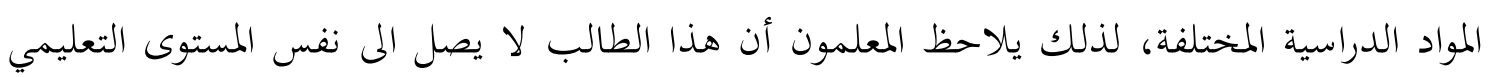

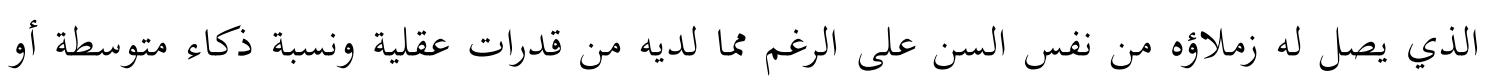
فوق المتوسطة.

$$
\begin{aligned}
& \text { ثانيا: أنواع صعوبات تعلمه اللغة العربية } \\
& \text { 1. صعوبات تعلم نمائية: }
\end{aligned}
$$

وهي تتعلق بنمو القدرات العقلية والعمليات المسئولة عن التوافق الدراسي للطالب البي

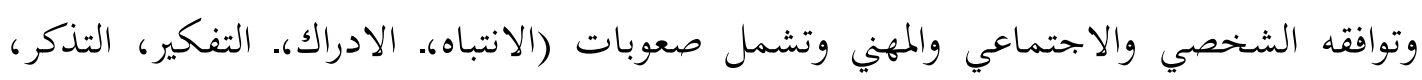

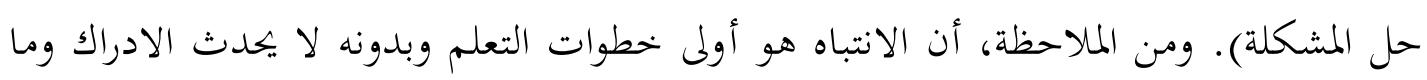

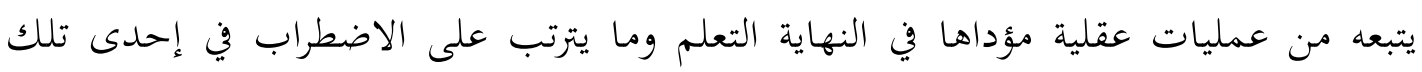
العمليات من انخفاض مستوى الطالب في المواد الدراسية المرتبطة بالقراءة والكتابة وغيرها.

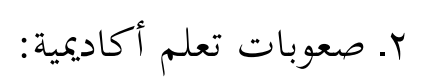

وهي تشمل صعوبات القراءة والكتابة والحساب، وهي نتيجة ومحصلة لصعوبات التعلم

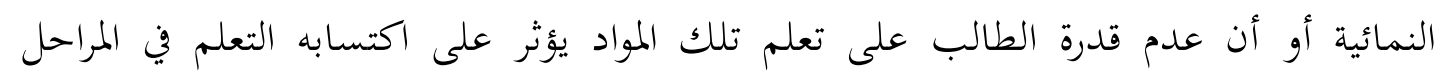
التالية. ثالثا: محاكات التعرف على صعوبات تعلم اللغة العربية. جدير بالمعرفة، أن هناك خمسة محكات يمك ن بها تحديد صعوبات التعلم والتعرف عليها وهي:

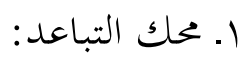

" ، عبد الحميد عبد الله وناصر عبد الله الغالى، أسس إعداد الكتب التعليمية لغير الناطقين بالعربية، دار الغالى، الرياض، المملكة العربية السعودية، ص: 
ويقصد به تباعد المستوى التحصيلي للطالب في مادة عن المستوى المتوقع منه حسب حالته

وله مظهران:

أ/ التفاوت بين القدرات العقلية للطالب والمستوى التحصيلي.

ب/ تفاوت مظاهر النمو التحصيلي للطالب في المقررات أو المواد الدراسية.

فقد يكون متفوقا في الرياضيات عاديا في اللغات ويعاني صعوبات تعلم في العلوم أو الدراسات الاجتماعية، وقد يكون التفاوت في التحصيل بين أجزاء مقرر دراسي واحد. ففي اللغة العربية مثلا، قد يكون طلق اللسان في القراءة جيدا، ولكنه يعاني صعوبات في استيعاب دروس النحو أو حفظ

$$
\text { r. النصوص الأدبية. }
$$

حيث يستبعد عند التشخيص وتحديد فئة صعوبات التعلم في الحالات الآتية: "التخلف العقلي، والاعاقات الحسية، والمكفوفين، وضعاف البصر، والصم، وضعاف السمع". وكذلك

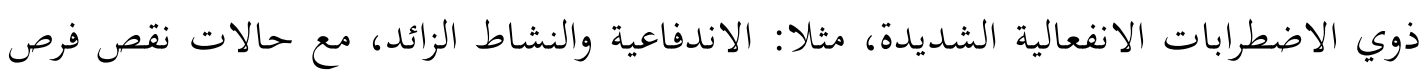

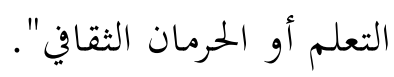

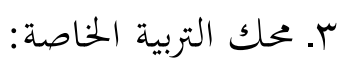

ويرتبط بالمحك السابق ومفاده أن ذوي صعوبات التعلم لا تصلح لمم طرق التدريس المتبعة مع الطلاب العاديين فضلا عن عدم صلاحية الطرق المتبعة مع المعاقين، وإنما يتعين توفير لون من صن التربية الخاصة من حيث التشخيص، والتصنيف، والتعليم، يختلف عن الفئات السابقة. ع. محك المشكلات المرتبطة بالنضوج:

حيث بند معدلات النمو تختلف من طالب لآخر محا يؤدي الى صعوبة هميئته لعمليات التعلم، فما هو معروف أن الطلاب الذكور يتقدم نموهم بمعدل إبطأ من الإناث مما يجعلهم في حوالي الخامسة أو السادسة غير مستعدين أو مهيئين من الناحية الإدراكية لتعلم التمييز بين الحروف الهجائية قراءة وكتابة مما يعوق تعلمهم اللغة. ومن ثم يتعين تقديم برامج تربوية تصحح قصور النمو الذي يعوق عمليات التعلم سواء كان هذا القصور يرجع لعوامل وراثية أو تكوينية أو بيئية. هـ محك العلامات الفيورولوجية: حيث يمكن الاستدلال على صعوبات التعلم من خلال التلف العضوي البسيط في المخ الذي يمكن فحصه من خلال رسام المخ الكهربائي، وينعكس الاضطراب البسيط في وظائف المخ في الاضطرابات الادراكية (البصري والسمعي والمكاني، النشاط الزائد 
والاضطرابات العقلية، صعوبة الاداء الوظيفي). وجديرا بالذكر، أن الاضطرابات في وظائف المخ ينعكس سلبيا على العمليات العقلية مما يعوق اكتساب الخبرات التربوية وتطبيقها والاستفادة منها بل يؤدي الى قصور في النمو الانفعالي، والاجتماعي، ونمو الشخصية العامة.

أن التطوير الذي يلحق اللغة العربية يكون في المحتوى اللغوي الذي يملأ القوالب النحوية والصرفية والصوتية، فالكلمات والعبارات والجمل (المحتوى) غير محدودة، وأما النظم الصوتي والصرفي والنحوي أها محدودة جداً، وأما التطور يكون في منهجية وطريقة توصيل هذه النظم للواقع التعليمي بطريقة مباشرة مناسبة لمستوى الدارسين على اختلاف مستوياهم. إن المفردات اللغوية تعد صورة لنوع الحضارة التي تتميز بها الأمة الناطقة بهذه اللغة، وهي خير دليل على مدى سعة خبراتما وعمقها، فحينما تكون خبرات الأمة محدودة، تأتي مفردات لغتها قليلة العدد، ضيقة الدلالة، وحينما تتسع خبرات الأمة، وتنضج حضارتا، تتسع لغتها تبعا لذلك، فتزداد ثروها اللفظية، وتتعدد دلالاتها. واللغة العربية تحمل كل هذه الميزات التي تميزها من غيرها من اللغات، وتطبيق المنهج التقابلي في الدراسة كان معينا على كشف الكثير من الصعوبات الصوتية التي تمثل أكثر الصعوبات النطقية لدى الدارسين للغة العربية من غير الناطقين بها. وقد تمت هذه الدراسة في البحث بالمقابلة بين العربية والإبحليزية مثالا تحليليا. وتحليل أخطاء الدارسين عند تعلمهم اللغة العربية أولا بأول مؤشر للتعرف على الصعوبات، سواء على المستوى الصوتي أو اللفظي أو النحوي أو الصرفي. فهذه الأخطاء إما داخل اللغة وإما أخطاء تطورية (Developmental) 


\section{المراجع}

ا ـ أحمد شلبب، "تعليم اللغة العببية لغير العرب" ، مكتب النهضة المصرية، القاهرة • 19 1. r. أحمد الشلبي- موسوعة التاريخ الإسلامي والحضارة الإسلامية- مكتبة النهضة العربية- الطبعة الأوليسنة سمو ام- القاهرة.

r. حلمي أحمد الوكيل، تطوير المناهج، مكتبة الأنحلو المصرية، القاهرة، بط، بم9 ام ع. ـ محادة إبراهيم، الابحاهات المعاصرة في تدريس اللغة العربية واللغات الحية الأخرى لغير الناطقين بها، دار الفكر العربي، القاهرة، 9^V أم. ه. . رشدي أحمد طعيمة، "تعليم اللغة العبية لغير الناطقين جها، مناهجه، وأساليبه" ، منشورات المنظمة الإسلامية للتربية والعلوم والثقافة، الرباط، 9 و أم.

7. . عبد الحميد عبد الله وناصر عبد الله الغالى، أسس إعداد الكتب التعليمية لغير الناطقين بالعبية، دار

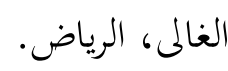

V. عبد الرحمن كادوك، تكنولوجيا التعليم: الماهية والأسس والتطبيقات العملية، دار المفردات للنشر لريصر

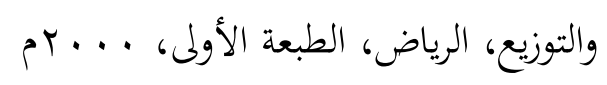

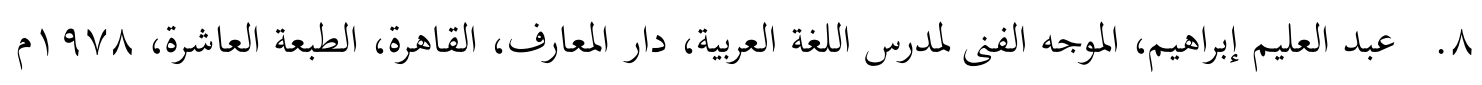
9. علي القاسم، الكتاب المدرسي لتعليم العربية لغير الناطقين بها، في السجل العامي للندوة العالمية الأولى لتعليم العربية لغير الناطقين بها: الجزء الثاني، جامعة الرياض، الرياض، · م9 ام • ا. عمر الصديق عبد الله، تعليم اللغة العربية للناطقين بغيرها: الطرق-الأساليب-الوسائل ، الدار العالمية للنشر والتوزيع، الهرم، الطبعة الاولى، م . . بام. الـ فتحي على يونس ومحمد عبد الرؤوف الشيخ، "المرجع في تعليم اللغة العبية للأجانب: من النظرية إلى التطبيق" ، مكتبة وهبة، القاهرة، سب ع اهـ/r . . ب م.

ז 1 ـ محمد على الخولي، "أساليب تلدرسي اللغة العربية" ، الطبعة الثالثة، بدون مطبعة، 919 ام. با ـ محمود كامل الناقة ورشدي أحمد طعيمة، "الكتاب الأساسي لتعليم اللغة العربية للناطقين بلغات

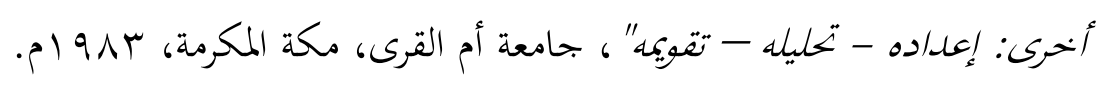

